UCRL-JC-133012

Preprint

\title{
Phase Segregation via Vlasov-Boltzmann Particle Dynamics
}

\author{
S. Bastea
}

This paper was prepared for submittal to 1998 Conference on Computational Physics

Granada, Spain

Sept. 2-5, 1998

January 19, 1999

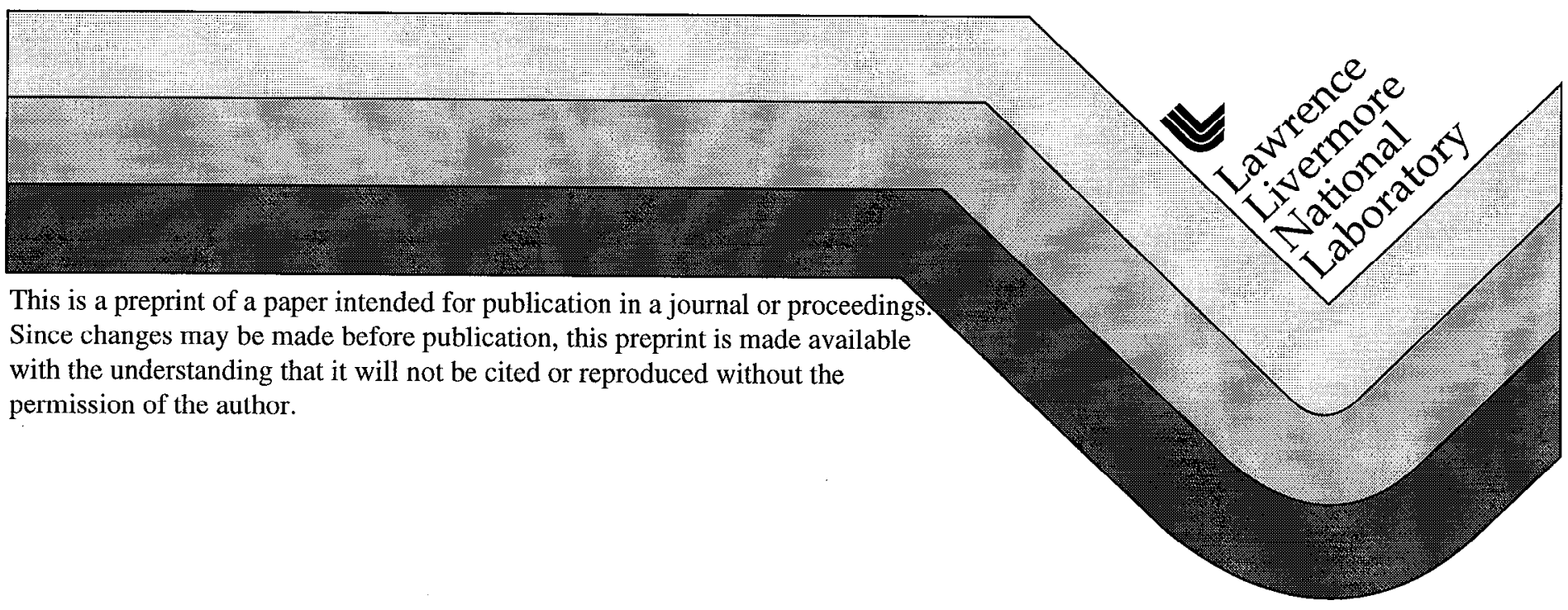




\section{DISCLAIMER}

This document was preparcd as an account of work sponsorcd by an agency of the United Statcs Government. Neither the United States Government nor the University of California nor any of their employees, makes any warranty, express or implied, or assumes any legal liability or responsibility for the accuracy, completencss, or uscfulness of any information, apparatus, product, or process disclosed, or represents that its use would not infringe privately owned rights. Reference herein to any specific commercial product, process, or service by trade name, trademark, manufacturer, or otherwise, does not necessarily constitute or imply its endorsement, recommendation, or favoring by the United States Government or the University of California. The views and opinions of authors expressed herein do not necessarily state or reflect those of the United States Government or the University of California, and shall not be used for advertising or product endorsement purposes. 


\title{
Phase Segregation via Vlasov-Boltzmann Particle Dynamics
}

\author{
S. Bastca \\ L-415, Lawrence Livermore National Laboratory \\ 7000 East Avenue, Livermore, CA 94550
}

The influence of hydrodynamics on phase segregation is an issue of both theoretical and practical importance that has been under intense scrutiny in recent years. In order to better understand and model the phase segregation of binary fluids we opted for a mesoscopic description that proves to be simplifying both conceptually and computationally [1].

The system that we studied is a mixture of two kinds of particles. All particles interact with each other through strong short-range interactions modeled by hard spheres with the same mass $m$ and diameter $d$. There is also a smooth long-range repulsion between particles of different kinds, $V(r)=\gamma^{3} U(\gamma r), \gamma>0 ; V(r)$ is a so-called Kac potential, $\int V(r) d^{3} \mathbf{r}=$ $\alpha>0$, that has the range and strength controlled by $\gamma$. This system is well described by mean-field theory and is known to undergo a phase transition to an immiscible state at low enough temperatures [2]. At low overall densities and weak enough repulsion the natural dynamical description for this system is given in terms of two coupled, energy and momentum conserving VlasovBoltzmann equations, making it what we call a dynamical mean-field model:

$$
\frac{\partial f_{i}}{\partial t}+\mathbf{v} \cdot \frac{\partial f_{i}}{\partial \mathbf{r}}+\frac{\mathbf{F}_{i}}{m} \cdot \frac{\partial f_{i}}{\partial \mathbf{v}}=J\left[f_{i}, f_{1}+f_{2}\right] \quad i=1,2
$$

$f_{i}(\mathbf{r}, \mathbf{v}, t)$ are the one-particle distribution functions, $\mathbf{F}_{i}(\mathbf{r}, t)=-\nabla V_{i}(\mathbf{r})$, $V_{i}(\mathbf{r})=\int V(|\mathbf{r}-\mathbf{r} \prime|) n_{j}(\mathbf{r} \prime) d \mathbf{r} \prime$ (Vlasor potential), $n_{j}(\mathbf{r} \prime)=\int f_{j}(\mathbf{r} \prime, \mathbf{v}, t) d \mathbf{v}$, with $i \neq j$, and $J[f, g]$ is the Boltzmann collision operator for hard core interactions [3]. The Boltzmann equation properly describes the dynamics of dilute gases, while the Vlasov term takes into account the long-range interaction in the spirit of the mean-field approximation: each particle moves in the background potential generated by all the other particles it is interacting with. Such a description is not only clarifying from a physical viewpoint, 
but opens the door for efficiently simulating the dynamics of the system at the particle level. We believe that this model contains the essential features of phase segregation in general binary fluids.

The computational scheme that we used is a combination of direct simulation Monte Carlo (DSMC) and particle-in-the-cell (PIC) evolution, that inherits the efficiency and robustness of these two algorithms. The DSMC is a stochastic algorithm due to Bird [4] that consistently incorporates the assumptions behind the Boltzmann equation into the particle dynamics. The method is essentially the following: the physical space is divided into a network of cells containing typically tens of particles and the free flow of the particles over a small time interval $\Delta t$ is followed by representative collisions among pairs of particles sharing the same cell. The typical linear dimension of a cell is a fraction of the mean free path between collisions.

The PIC method for integrating the equations of motion was first used to deal with the $1 / r$ potential in plasma physics [5]. It takes advantage of the simple form of the Vlasov potential, which is a product in Fourier space, by calculating the densities on a grid through some weighting, then the potentials and forces on the same grid, and finally interpolating the forces at the position of each particle.

These two methods can be naturally brought together by replacing the free flow of the DSMC procedure by motion in the background, Vlasov potential. If the repulsive potential between the two species is sufficiently wcak and long ranged (so no new inter-particle correlations are introduced), such an algorithm contains the essential ingredients of the Vlasov-Boltzmann kinetics, and is therefore physically consistent with Eq. 1. To this end we used a leap-frog integrator for the equations of motion:

$$
\begin{gathered}
\mathbf{r}(t+\Delta t)=\mathbf{r}(t)+\mathbf{v}\left(t+\frac{\Delta t}{2}\right) \Delta t \\
\mathbf{v}(t)=\mathbf{v}\left(t-\frac{\Delta t}{2}\right)+\frac{\mathbf{f}(t)}{m} \frac{\Delta t}{2} ; \mathbf{v}\left(t+\frac{\Delta t}{2}\right)=\mathbf{v} \prime(t)+\frac{\mathbf{f}(t)}{m} \frac{\Delta t}{2}
\end{gathered}
$$

with the DSMC collisions (note $\mathbf{v}$ ) taking place at time $t$. The natural units for lengths and times in this method are the mean-free path, $\lambda=\left(2^{\frac{1}{2}} \pi n d^{2}\right)^{-1}$, and mean-free time, $\tau=\lambda / c$, where $n$ is the overall particle density, $c=\left(2 k_{B} T_{0} / m\right)^{\frac{1}{2}}$ and $T_{0}$ is the (initial) tcmperature. The DSMC requires that the system is dilute, i.e. $n d^{3} \ll 1$, which in turn means that $n \lambda^{3}$ is large, while for lhe Vlasov calculation we need to have a significant number of particles in the 'sphere of influence' of the potential, roughly meaning 


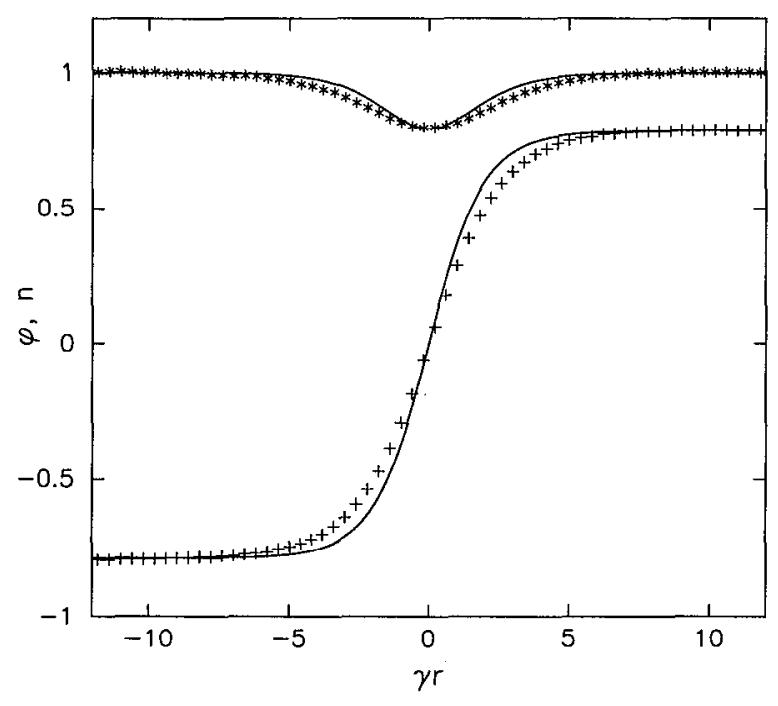

Figure 1: Equilibrium interface profile: $n(+)$ is the normalized total particle density and $\varphi(*)$ is the concentration difference between the two species, $\varphi=\left(n_{1}-n_{2}\right) /\left(n_{1}+n_{2}\right)$. The symbols are the results of simulations $(\gamma=2)$ and the lines are the solution of the Vlasov-Boltzmann equations.

$n \gamma^{-3} \gg 1$. We would also like to have a potential range that is much smaller than the system size, so that we can efficiently study the phase separation kinetics inside the miscibility gap. The idea is that we want to be able to follow the growth of the domains of the two phases for a long time, particularly in the regime where the interface between phases has a thickness much bigger that the domain size. Because far away from the critical temperature the interface thickness is of the same order as $\gamma^{-1}$, the system must be large enough in units of $\gamma^{-1}$. All these requirements can generally be met with reasonable computational resources. For example, we were able to perform simulations with over 2 million particles on a DEC Alpha station with 256 $\mathrm{MB}$ of memory. To insure the stability of the algorithm the potential grid should be fine enough, but more important is the use of quadratic spline interpolation for the grid quantities and a ten-point finite difference method for the forces [5]. This scheme insures very good conservation of the total energy and linear momentum with time steps as large as $0.1-0.2 \tau$.

The structure of the interface separating the two phases coexisting inside the miscibility gap is related to the dominating coarsening mechanism [6]. We compared the equilibrium interface profiles that result directly from the 


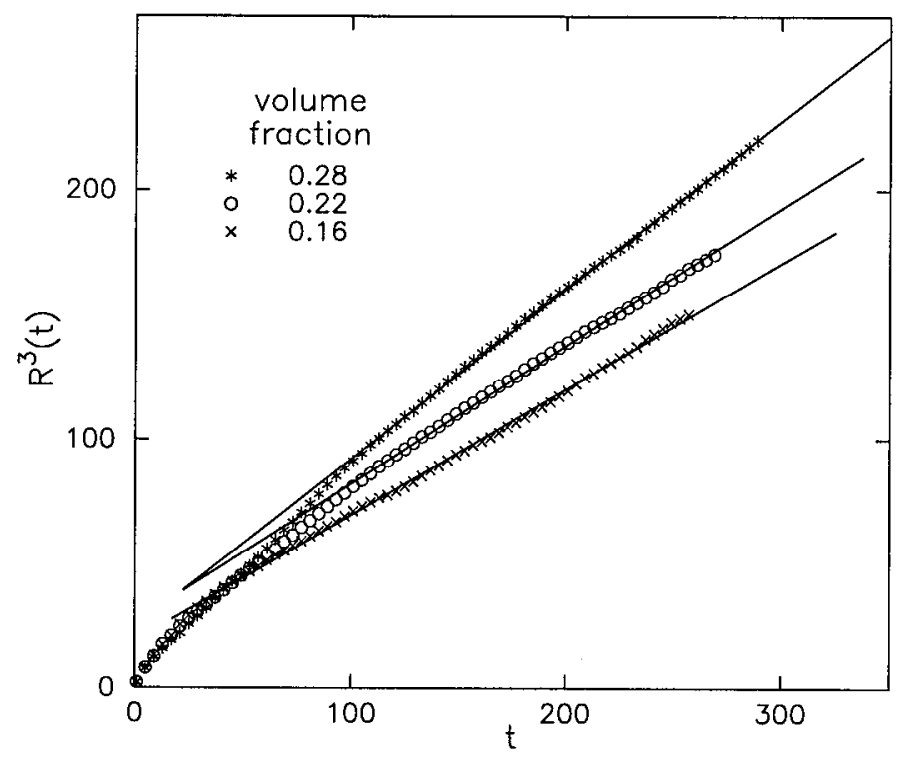

Figure 2: Domain growth for off-critical quenches: $R(t)$ is the typical domain size at time $t$; straight line fits are drawn.

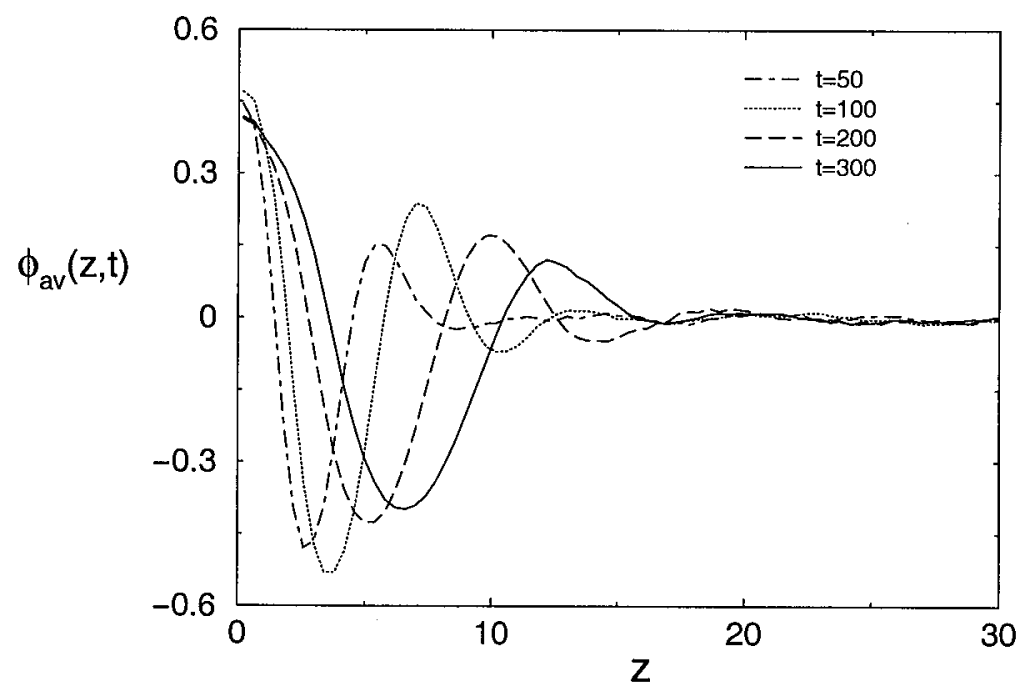

Figure 3: Order parameter (concentration difference) averaged in slices parallel to the attracting wall as a function of the distance to the wall; nonwetting case. 


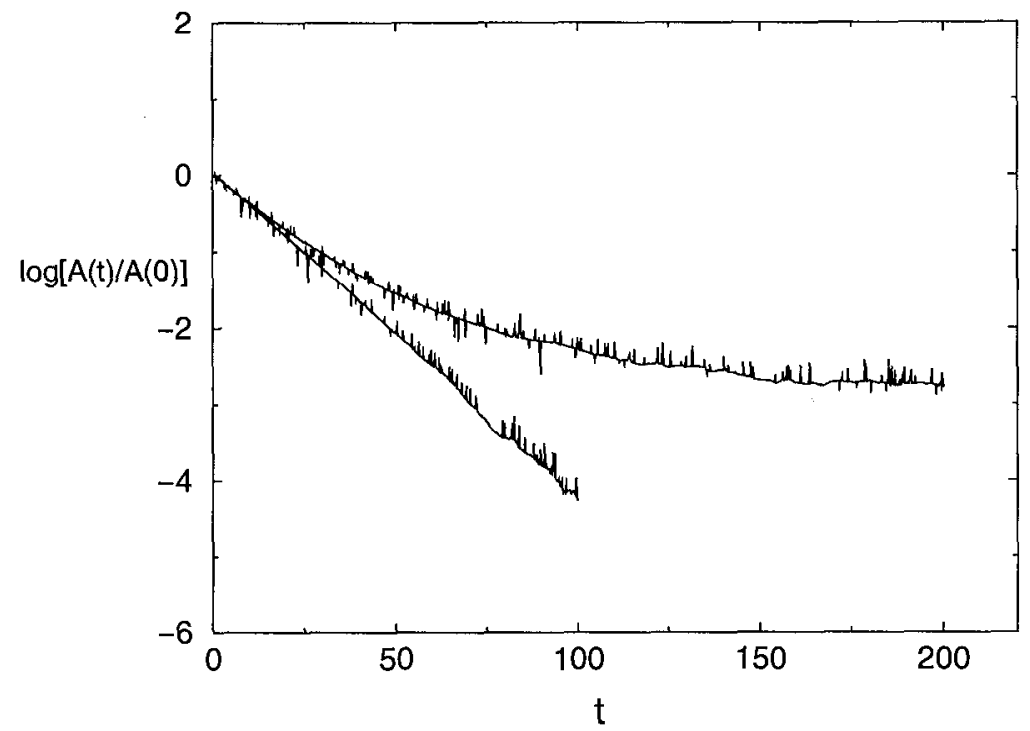

Figure 4: Decay of a cosine velocity profile (see text) for $T / T_{c}=1.5$ (bottom) and $T / T_{c}=0.5$ (top); the system is initially homogeneous.

Vlasov-Boltzmann equations [1] with the profiles obtained in simulations, Fig. 1, and found very good agreement. The agreement further improves as $\gamma$ is decreased and we conclude that the computational method is consistent with the Vlasov-Boltzmann kinetics, Eq. 1. Simulations performed at various volume fractions of the two coexisting phases provide a detailed picture of phase segregation in fluid systems with momentum conservation (model $\mathrm{H})$ and without externally induced flows [1]. In particular, we found that for off-critical quenches but rather large volume fractions of the two phases the growth of the domains proceeds at late times as $t^{\frac{1}{3}}$, Fig. 2, a result first observed in microgravity experiments [7], that we attributed to the so-called Brownian droplet coalescence mechanism.

The influence of a wall attracting preferentially one of the species on the phase segregation process has drawn much attention in recent years [8]. Typically, phase separation waves propagate into the bulk perpendicular to the surface, with the resulting anisotropic pattern and growth exponents depending on whether or not the preferred component wets the surface. The present method is a useful tool for studying this process in binary fluids, Fig. 3 , which have been studied mainly experimentally.

Our model and computational scheme provide a convenient framework 
for the study of another important problem, the influence of phase segregation on an initially prescribed hydrodynamical flow. A simple relevant case is the following: the system is homogeneous at $t=0$ with equal numbers of the two types of particles and we prescribe a velocity profile on a 'macroscopic' scale, $v_{x}=A(0) \cos (k y)\left(k=\frac{2 \pi}{L}, L\right.$ is the length of the system along $y$ ), with a small initial amplitude $A(0)$. The time evolution of this profile is very different above and below the critical temperature, $T_{c}$. In the homogeneous region the shape of the profile is preserved and the amplitude $A(t)$ decays exponentially, $A(t)=A(0) \exp \left(-k^{2} \nu t\right)$, similarly to the case with no long range repulsions ( $\nu$ is the kinematic viscosity of the Boltzmann gas), while below $T_{c}$ the system segregates and $A(t)$ decays slower than exponentially, Fig. 4. (Note that the $T^{\frac{1}{2}}$ dependence of the viscosity [3] has been scaled out.)

The approach to phase segregation kinetics described here takes advantage of an important analytical tool available in nonequilibrium physics, the Boltzmann equation, and has a computational simplicity that should make it uscful for other interesting applications.

\section{References}

[1] S. Bastea and J.L. Lebowitz, Phys. Rev. Lett. 78, 3499 (1997); S. Bastea, R. Esposito, J.L. Lebowitz, R. Marra, in preparation.

[2] J.L. Lebowitz and O. Penrose, J. Math. Phys.(N.Y.) 7, 98 (1966); J.L. Lebowitz and E.H. Lieb, Phys. Lett. 39A, 98 (1972).

[3] S. Chapman and T.G. Cowling, The Mathematical Theory of Nonuniform Gases, (Cambridge University Press, 1970).

[4] G.A. Bird, Molecular Gas Dynamics, (Clarendon Press, Oxford, 1976).

[5] C.K. Birdsall and A.B. Langdon, Plasma Physics Via Computer Simulation, (McGraw-Hill Book Company, New York, 1985).

[6] E.D. Siggia, Phys. Rev. A 20, 595 (1979).

[7] F. Perrot, P. Guenoun, T. Baumberger and D. Beysens, Phys. Rev. Lett. 73, 688 (1994).

[8] S. Puri, K. Binder, H.L. Frisch, Phys. Rev. E 56, 6991 (1997); S. Bastea, S. Puri, J.L. Lebowitz, in preparation. 